\title{
ChemComm
}

Check for updates

Cite this: Chem. Commun., 2022, 58,1330

Received 5th November 2021, Accepted 22nd December 2021

DOI: $10.1039 / \mathrm{d} 1 \mathrm{cc} 06250 f$

rsc.li/chemcomm

\section{A facile and sustainable one-pot approach to the aqueous and low-temperature PET-to-UiO-66(Zr) upcycling $\dagger$}

\author{
Maria Crespo Ribadeneyra, ${ }^{\mathrm{a}}$ James King, ${ }^{\mathrm{b}}$ Maria Magdalena Titirici (D) ${ }^{\mathrm{a}}$ and \\ Petra Ágota Szilágyi (iD) *b
}

\begin{abstract}
Accelerating waste management requires the conversion of polymer waste to value-added materials through sustainable approaches. While depolymerised PET has been used as feedstock to produce metal-organic frameworks, this is the first report of the successful one-pot hydrothermal synthesis of the desirable UiO-66 topology through the judicious choice of reactants, modulators and reaction conditions.
\end{abstract}

The current level of plastic production exceeds 300 MT worldwide and it is set to increase even further. ${ }^{1}$ After the first use, most plastics are discarded to landfills or incinerated, while only $9 \%$ are recycled. Almost half of the discarded plastic consists of single-use polyolefin-based packaging. ${ }^{2}$ Among these, PET (polyethylene terephthalate), used for plastic bottles and 'polyester' fibres, is discarded at the highest rate and contributes to $7.5 \%$ of the total global plastic waste. ${ }^{3,4} \mathrm{PET}$ is fully recyclable, yet recycling rates remain low in most countries, including the USA, where it reaches only around $30 \% .^{5}$ It has been shown that recycling PET allows for about $20 \%$ reduction of the greenhouse gas emissions associated with the manufacturing process when compared with their virgin (fossil-fuel derived) counterpart, while life-cycle water consumption has been found to be similar for the recycled and virgin plastics. ${ }^{6}$ However, this does not account for the upfront cost of a recycling plant and additional costs incurring on selection and distribution. For this reason, the upcycling of PET waste, i.e. its conversion into value-added products is an attractive approach, which has received significant interest in the textile industry in particular. ${ }^{7}$

\footnotetext{
${ }^{a}$ Department of Chemical Engineering, Imperial College London,

South Kensington Campus, London, SW7 2AZ, UK

${ }^{b}$ School of Engineering and Materials Science, Queen Mary University of London,

Mile End Road, London E1 4NS, UK. E-mail: p.szilagyi@qmul.ac.uk

$\dagger$ Electronic supplementary information (ESI) available: Experimental conditions, PXRD of all samples and reference materials, XPS spectra and fitting, TGA and DSC curves. See DOI: 10.1039/d1cc06250f
}

It should be emphasised that the monomers of PET, in particular terephthalic acid, are of great importance to the chemical industry as well, and it is one of the most prevalent building block of metal-organic frameworks (MOFs).

MOFs are a class of hybrid materials built up of inorganic nodes interconnected by organic linkers. From a topological viewpoint, we may distinguish two kinds of MOFs built up of single-cation or oxide-cluster nodes; in these two cases, the central metal cations have significantly different coordination environments resulting in different flexibility, e.g. breathing effect in MIL-53 - based on single cations, ${ }^{8}$ and pre-requisites for framework self-assembly, e.g. the necessity of the formation of hexanuclear $\mathrm{Zr}$ complexes to assemble the UiO-66 topology. ${ }^{9,10}$

MOFs boast exciting and unique properties, such as chemical and structural modularity, enhanced and exploitable through their high porosity and crystallinity, leading to a host of possible applications ranging from catalysis, gas storage and separation, sensing, energy storage, drug delivery, etc. ${ }^{11}$ However, MOF syntheses often require expensive reactants and harsh conditions, which makes them costly both economically and ecologically. Incorporating waste-derived reactants in their synthesis would make their production both greener and cheaper, thereby potentially enabling their applications.

In this perspective, the upcycling of PET into MOFs is an attractive concept, which has been explored recently. First demonstrations of PET-to-MOF upcycling made use of an indirect approach consisting of the initial depolymerisation of PET yielding terephthalic acid (1,4-benzenedicarboxylic acid, BDC), subsequent purification, and MOF synthesis according to conventional solvothermal routes. Examples include $\mathrm{Cu}(\mathrm{BDC})^{12}$ and UiO-66(Zr), ${ }^{13}$ with single cation and oxide-cluster inorganic nodes, respectively. It is important that any process used in the PET upcycling be as sustainable as possible to avoid harmful and energy-demanding processes. In fact, green chemical synthetic principles should be applied whenever possible. In this vein, De Vos et al. ${ }^{14}$ developed a one-pot hydrothermal route for direct PET-to-MOF conversion, an approach which was also successfully applied to the synthesis of 
other MOFs based on single-cation nodes (e.g. MIL-53(Al/Cr/Ga), MIL-47(V), and more recently $\left.\mathrm{Ca}(\mathrm{BDC})\left(\mathrm{H}_{2} \mathrm{O}\right)_{3}\right) \cdot{ }^{14-16}$ Despite the above advances however, one-pot PET-to-MOF upcycling processes to frameworks with oxide-cluster nodes have not been developed successfully under hydrothermal conditions. In fact, the desirable UiO-66 topology has only been obtained in its highly defectful and thus less stable $h c p$ polymorph and only under solvothermal conditions. $^{17}$

In fact, to date, no reports have been published of direct PET-to-MOF conversion in aqueous environment under mild conditions, particularly when the MOFs' inorganic nodes are complex oxide-like clusters, such as in the case of the UiO-66 topology. UiO-66(Zr) topology of MOFs is highly interesting as these frameworks are stable, crystalline and have been demonstrated to be beneficial in a variety of applications. ${ }^{18}$ As such, finding approaches for its sustainable production, i.e. in aqueous media using plastic waste as precursors, is very important for enabling its real-life applications.

Some of us have been previously developing understanding on the conditions necessary for the formation of the node precursor enabling self-assembly in aqueous conditions, which was found to depend on the precursors, modulators, $\mathrm{pH}$, etc. ${ }^{19,20}$ Using these results and in order to develop a facile, one-pot procedure for the upcycling of PET to UiO-66(Zr) under mild hydrothermal conditions, we have designed a matrix of various parameters influencing the formation of the inorganic node - crucial to the self-assembly of the MOF. We selected two different inorganic precursors with significantly different solubility in water: $180 \mathrm{mg}$ anhydrous $\mathrm{ZrCl}_{4}$ and $95 \mathrm{mg} \mathrm{ZrO}_{2}$. As the acid catalyst for the PET depolymerisation reaction we used $1.8 \mathrm{ml} c c \mathrm{HNO}_{3}$ or $0.4 \mathrm{ml} c c \mathrm{HCl}$ combined with $0.1 \mathrm{ml}$ glacial acetic acid modulator. $300 \mathrm{mg}$ PET linker feedstock was compared with $130 \mathrm{mg}$ terephthalic acid (BDC). All reagents were purchased from Sigma Aldrich and used without further purification. In all cases $8 \mathrm{ml}$ deionised water was used as solution and reactions were conducted in scintillation vials at $80{ }^{\circ} \mathrm{C}$ for one week. The reaction mixtures were then filtered and washed with deionised water until the solutions were no longer acidic, as checked with the help of a pH paper. In Table 1, the combination of reactants and reagents are summarized.

To verify whether any transformation has taken place, we carried out Fourier-transformed Infrared spectroscopy (FTIR), while the MOF topology and phase were checked using powder $\mathrm{X}$-ray diffraction (PXRD).

Table 1 Summary of the reactants and reagents used in the PET-to-MOF upcycling reactions

\begin{tabular}{llll}
\hline Code & Inorganic & Organic & Acid \\
\hline OBN & $\mathrm{ZrO}_{2}$ & BDC & $\mathrm{HNO}_{3}$ \\
OBA & $\mathrm{ZrO}_{2}$ & BDC & $\mathrm{HCl} / \mathrm{Ac}$ \\
OPN & $\mathrm{ZrO}_{2}$ & PET & $\mathrm{HNO}$ \\
OPA & $\mathrm{ZrO}_{2}$ & PET & $\mathrm{HCl} / \mathrm{Ac}$ \\
ClBN & $\mathrm{ZrCl}_{4}$ & BDC & $\mathrm{HNO}$ \\
ClBA & $\mathrm{ZrCl}_{4}$ & BDC & $\mathrm{HCl} / \mathrm{Ac}$ \\
ClPN & $\mathrm{ZrCl}_{4}$ & PET & $\mathrm{HNO}$ \\
ClPA & $\mathrm{ZrCl}_{4}$ & PET & $\mathrm{HCl} / \mathrm{Ac}$
\end{tabular}
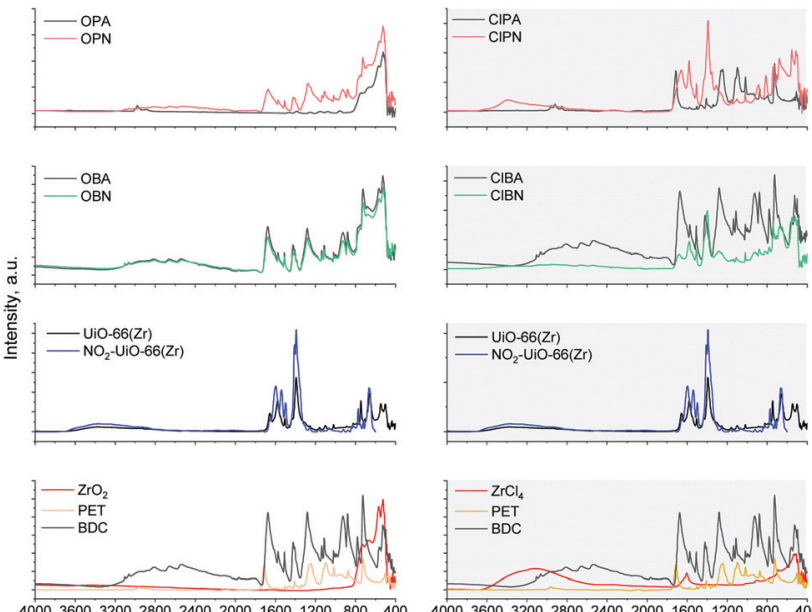

Wavenumber, $\mathrm{cm}^{-1}$

Fig. 1 FTIR spectra of the different reaction mixtures, conditions summarised in Table 1, and reference materials.

Previously, it has been suggested that the application of nitric acid for PET upconversion to the MIL-53 MOF resulted in the nitrolysis of the linker's aromatic rings. ${ }^{14}$ In order to account for this FTIR spectra and PXRD patterns were acquired for both the pristine UiO-66(Zr) and the $\mathrm{NO}_{2}$-UiO-66(Zr) for reference, both of which were synthesised solvothermally, according to the method published in ref. 21. In addition, FTIR and PXRD data were also collected on all reactants.

FTIR spectra of the as-synthesised mixtures and reference materials (Fig. 1) reveal that only in the case of ClPN and ClBN samples show significant conversions.

It should also be noted that in the case of the CIPN reaction mixture, the presence of new bands may be observed around $3500 \mathrm{~cm}^{-1}$, indicative of $\mathrm{N}-\mathrm{H}$ modes, which is in contrast to what was suggested previously, i.e. PET hydrolysis in nitric acid conditions leads to the formation of $\mathrm{NO}_{2}$ groups on the aromatic ring. ${ }^{14}$ We suggest that the ethylene glycol molecules resulting from the PET depolymerisation reaction are responsible for this reduction reaction, as observed previously. ${ }^{22}$

To verify if $\mathrm{N}-\mathrm{H}$ bonds indeed have been formed we have carried out X-ray photoelectron spectroscopy (XPS, Fig. S2-S4, $\mathrm{ESI} \dagger$ ). The main peak at $400.2 \mathrm{eV}$ corresponds very well with the $\mathrm{N}$ binding energy in $\mathrm{NH}_{2}$-UiO-66( $\left.\mathrm{Zr}\right),{ }^{23}$ with only a minor peak designating higher oxidation state (at $407.3 \mathrm{eV}$ ) accounting for only $c a .16 \%$ of the total of $\mathrm{N}$ content (ESI, $\dagger$ Table S1), which can be assigned to the more oxidised $-\mathrm{NO}_{2}$ groups. This means that the process predominantly yields amino-functionalised terephthalate rather than $\mathrm{NO}_{2}$-functionalised one, contrarily to what was presumed previously. The presence of $-\mathrm{NH}_{2}$ groups on the UiO-66(Zr) in effect translates to various advantages when it comes to potential applications ranging from improved $\mathrm{CO}_{2}$ uptake with respect to the pristine UiO- $66^{24}$ to its ability to embed catalytically active $\mathrm{Pd}$ nanoclusters inside its pores unlike the pristine UiO-66. ${ }^{23}$

However, it is not the mere establishment of whether PET conversion has taken place but also the establishment of 


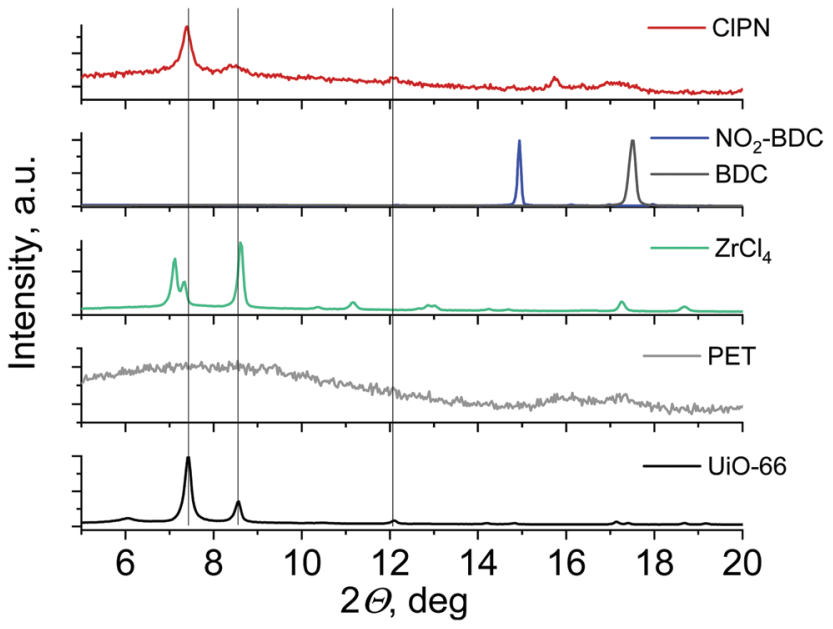

Fig. 2 PXRD traces of the product of the successfully converted PET-toMOF reaction mixture and reference materials. Vertical lines were included to guide the eyes.

whether the desired phase has been formed that is crucial. For this reason, we have collected PXRD data on the only sample (ClPN) in which conversion of PET to MOF has been observed (Fig. 2).

It is apparent that the resultant crystalline fraction is of the UiO-66 topology, while some amorphous contribution from the residual PET can also be observed. It is important to note that a significant excess $\left(0.77 \mathrm{mmol} \mathrm{ZrCl}_{4}\right.$ vs. $1.56 \mathrm{mmol}$ terephthalate) of PET was used for this reaction, so we estimate that the overall conversion rate is significant. Interestingly, the pattern obtained on the only other sample that showed a degree of conversion based on the FTIR results, ClBN, revealed UiO-66 topology formation to a much lower degree of crystallinity (Fig. S1, ESI $\dagger$ ). It should be noted that this conversion is not accompanied by the appearance of $\mathrm{N}-\mathrm{H}$ vibration modes in the FTIR spectrum, i.e. the terephthalate linker is not functionalised on conversion. This in turn would lead to lower linker solubility in aqueous medium and consequently to poorer conditions for the formation of crystallites with the UiO-66 topology. ${ }^{25}$

Interestingly, the PXRD pattern of the MOF obtained through PET upcycling appears to be less defective based; on carefully controlling defect concentration and structure it has been noted that missing cluster defects result in notable changes in the diffraction pattern, leading to the prevalence of the Reo phase. ${ }^{26}$ While some evidence of the Reo phase can be observed in the pattern of the solvothermally synthesised UiO-66 (Reo(110) at $\left.c a .6^{\circ}\right)$, such indication is apparently absent in the PET upcycled sample pattern.

As perhaps the most important property of a MOF is its porosity, which is also related to the quality of the produced crystallites significantly, ${ }^{27}$ we have also carried out specific surface area and porosity analysis. In addition to the textural analysis of the MOF as obtained by the upcycling of PET, we have also acquired data on the pristine and $\mathrm{NO}_{2}{ }^{-}$and $\mathrm{NH}_{2}{ }^{-}$ functionalised UiO-66(Zr) synthesised by solvothermal method
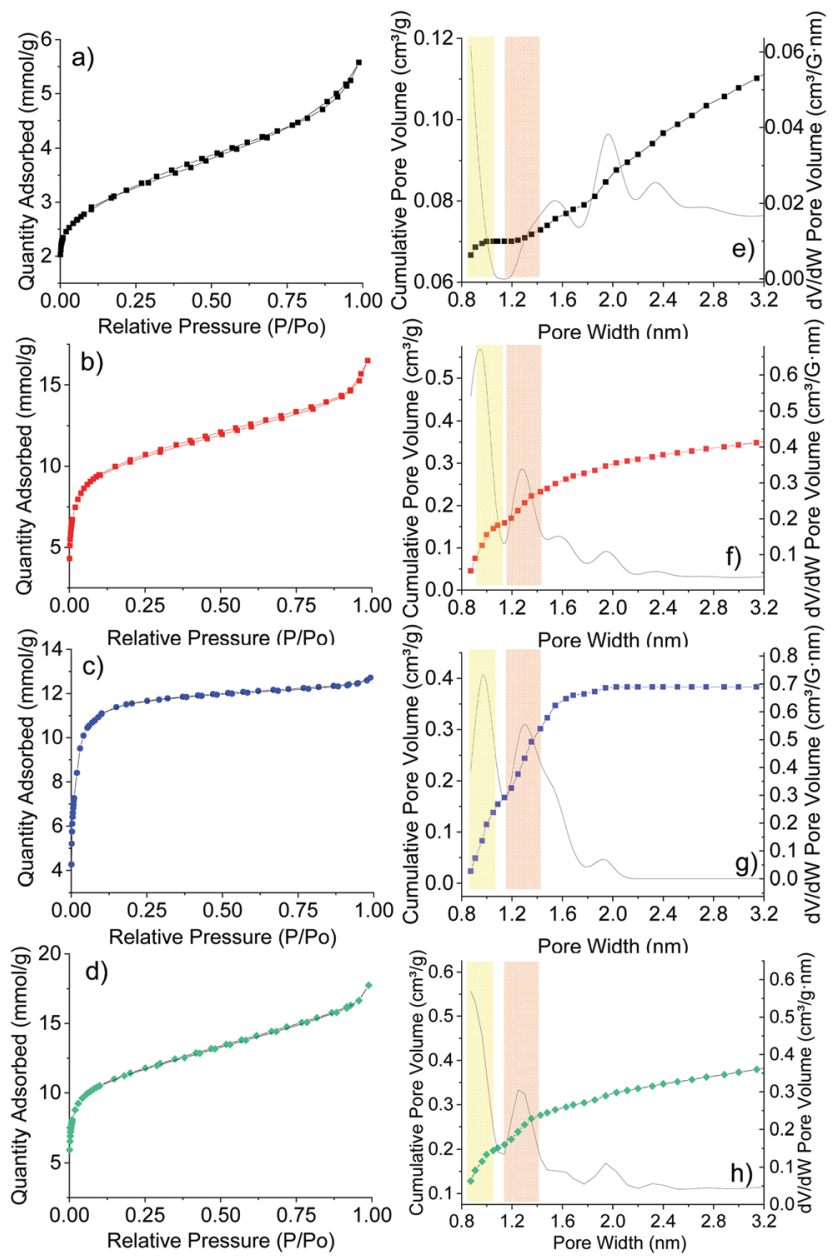

Fig. $3 \quad \mathrm{~N}_{2}$ adsorption isotherms $(a-d)$ and pore widths (e-h) of the upcycled PET-to-UiO-66(Zr) sample (a and e) and reference samples $\mathrm{NO}_{2}$-UiO-66(Zr) (b and g), UiO-66 ( $\mathrm{Zr}$ ) (c and g), and $\mathrm{NH}_{2}-\mathrm{UiO}-66(\mathrm{Zr}$ ) ( $\mathrm{d}$ and h).

for reference. The data for the reference MOFs is also displayed in Fig. 3 for comparison.

Two significant conclusions may be formed from the analysis of these datasets. On the one hand, the BET surface area of the MOF derived from PET is smaller than that of the functionalised MOF by $c a$. a factor of $3\left(251 \mathrm{~m}^{2} \mathrm{~g}^{-1}, 848 \mathrm{~m}^{2} \mathrm{~g}^{-1}\right.$ for the $\mathrm{NO}_{2}$-UiO-66, and $929 \mathrm{~m}^{2} \mathrm{~g}^{-1}$ for the $\mathrm{NH}_{2}$-UiO-66, respectively). We can assign this apparent reduction tentatively to the presence of PET in the sample, as already observed in the PXRD pattern (Fig. 2). Unfortunately, our attempts to better quantify the conversion rates using thermal analyses were unsuccessful on account of (i) the existence of an apparent UiO-66 fraction in addition to the functionalised reaction products (additional decomposition step with onset temperature of $c a .400{ }^{\circ} \mathrm{C}$, Fig. S5a, ESI $\dagger$ ) and (ii) the critical changes in the residual PET properties (the disappearance of the most significant endothermic peak at $c a .200{ }^{\circ} \mathrm{C}$ on conversion, Fig. S5b, ESI $\dagger$ ), which we attribute to the chain shortening accompanying the upconversion. Nevertheless, we may estimate the PET conversion rate to be over $50 \%$ at such mild conditions, since the terephthalate excess was two-fold. 
Importantly, the pore diameters of the MOF obtained through PET conversion and that of the functionalised MOF highly accurately replicate the theoretical pore diameters of $7.5 \AA$ (in yellow) and $12 \AA$ (in coral) for the tetrahedral and octahedral pores, respectively, ${ }^{28}$ demonstrating the formation of the desired phase. The proportion of these pore widths with respect to each other and other pore sizes (of 16, 20, and $24 \AA$, $N . B$. these pore sizes can be assigned to merged pores due to missing linker effects) however is not the same, which can be an effect of the presence of the PET either as an adsorbent on its own or on the MOF-PET interface, or some possible defects. ${ }^{29}$ We would like to point out however that neither our PXRD nor our XPS data revealed a significant extent of defect formation in the upcycled sample. The effect of prevalent defect formation, leading to the Reo phase, on the porous structure is apparent when the datasets for the reference materials are compared, as such kind of defect in the pristine UiO-66(Zr) was observed with PXRD. In particular, the highresolution Zr3d XPS spectrum (Fig. S4, ESI $\dagger$ ) of the upcycled sample is practically identical to what was previously published. $^{30}$

In summary, we demonstrate that green chemical principles, i.e. mild hydrothermal conditions, may be applied to upcycle PET plastic waste into value-added functionalised metal-organic frameworks. When the conditions are judiciously selected, even MOFs requiring more challenging synthetic conditions on account of their inorganic node structures, i.e. oxide-cluster like, may be formed. Furthermore, the soobtained MOFs are formed in reasonable yield and without defect formation significant enough of leading to structural changes. We also note that the PET depolymerisation byproduct (ethylene glycol) acts as a reducing agent on the linker functionality, transforming $-\mathrm{NO}_{2}$ groups into $-\mathrm{NH}_{2}$. We anticipate therefore that the functional group space within MOFs may be further explored in such a one-pot approach in the future.

The authors thank the EPSRC for funding: EP/S018204/2 "Sustainable Processing of Energy Materials from Waste".

\section{Conflicts of interest}

There are no conflicts to declare.

\section{References}

1 H. Sardon and A. P. Dove, Science, 2018, 360, 380-381.

2 R. Geyer, J. R. Jambeck and K. L. Law, Sci. Adv., 2017, 3, e1700782.

3 G. Lopez, M. Artetxe, M. Amutio, J. Bilbao and M. Olazar, Renewable Sustainable Energy Rev., 2017, 73, 346-368.
4 U. S. Chaudhari, Y. Lin, V. S. Thompson, R. M. Handler, J. M. Pearce, G. Caneba, P. Muhuri, D. Watkins and D. R. Shonnard, ACS Sustainable Chem. Eng., 2021, 9, 7403-7421.

$5 \mathrm{M}$. Tsakona and I. Rucevska, Baseline report on plastic waste, Seychelles, 2020.

6 P. T. Benavides, J. B. Dunn, J. Han, M. Biddy and J. Markham, ACS Sustainable Chem. Eng., 2018, 6, 9725-9733.

7 S. H. Park and S. H. Kim, Fash. Text., 2014, 1, 1.

8 C. Serre, F. Millange, C. Thouvenot, M. Noguès, G. Marsolier, D. Louër and G. Férey, J. Am. Chem. Soc., 2002, 124, 13519-13526.

9 Z. Hu, I. Castano, S. Wang, Y. Wang, Y. Peng, Y. Qian, C. Chi, X. Wang and D. Zhao, Cryst. Growth Des., 2016, 16, 2295-2301.

10 C. Hennig, S. Weiss, W. Kraus, J. Kretzschmar and A. C. Scheinost, Inorg. Chem., 2017, 56, 2473-2480.

11 S. Yuan, L. Feng, K. Wang, J. Pang, M. Bosch, C. Lollar, Y. Sun, J. Qin, X. Yang, P. Zhang, Q. Wang, L. Zou, Y. Zhang, L. Zhang, Y. Fang, J. Li and H.-C. Zhou, Adv. Mater., 2018, 30, 1704303.

12 Manju, P. Kumar Roy, A. Ramanan and C. Rajagopal, Mater. Lett., 2013, 106, 390-392.

13 X. Dyosiba, J. Ren, N. M. Musyoka, H. W. Langmi, M. Mathe and M. S. Onyango, Ind. Eng. Chem. Res., 2019, 58, 17010-17016.

14 W. P. R. Deleu, I. Stassen, D. Jonckheere, R. Ameloot and D. E. De Vos, J. Mater. Chem. A, 2016, 4, 9519-9525.

15 S.-H. Lo, D. Senthil Raja, C.-W. Chen, Y.-H. Kang, J.-J. Chen and C.-H. Lin, Dalton Trans., 2016, 45, 9565-9573.

16 T. S. Crickmore, H. B. Sana, H. Mitchell, M. Clark and D. Bradshaw, Chem. Commun., 2021, 57, 10592-10595.

17 L. Zhou, S. Wang, Y. Chen and C. Serre, Microporous Mesoporous Mater., 2019, 290, 109674.

18 J. H. Cavka, S. Jakobsen, U. Olsbye, N. Guillou, C. Lamberti, S. Bordiga and K. P. Lillerud, J. Am. Chem. Soc., 2008, 130, 13850-13851.

19 I. Pakamore, J. Rousseau, C. Rousseau, E. Monflier and P. Á. Szilágyi, Green Chem., 2018, 20, 5292-5298.

20 G. Hoyez, J. Rousseau, C. Rousseau, S. Saitzek, J. King, P. Á. Szilágyi, C. Volkringer, T. Loiseau, F. Hapiot, E. Monflier and A. Ponchel, CrystEngComm, 2021, 23, 2764-2772.

21 M. J. Katz, Z. J. Brown, Y. J. Colón, P. W. Siu, K. A. Scheidt, R. Q. Snurr, J. T. Hupp and O. K. Farha, Chem. Commun., 2013, 49, 9449-9451.

22 S. Chandrasekhar, S. J. Prakash and C. L. Rao, J. Org. Chem., 2006, 71, 2196-2199.

23 D. E. Coupry, J. Butson, P. S. Petkov, M. Saunders, K. O’Donnell, H. Kim, C. Buckley, M. Addicoat, T. Heine and P. Á. Szilágyi, Chem. Commun., 2016, 52, 5175-5178.

24 I. Strauss, K. Chakarova, A. Mundstock, M. Mihaylov, K. Hadjiivanov, N. Guschanski and J. Caro, Microporous Mesoporous Mater., 2020, 302, 110227.

25 D. Bůžek, S. Adamec, K. Lang and J. Demel, Inorg. Chem. Front., 2021, 8, 720-734.

26 P. Chammingkwan, G. Y. Shangkum, L. T. T. Mai, P. Mohan, A. Thakur, T. Wada and T. Taniike, $R S C A d v$. , 2020, 10, 28180-28185.

27 S. Øien-Ødegaard, G. C. Shearer, D. S. Wragg and K. P. Lillerud, Chem. Soc. Rev., 2017, 46, 4867-4876.

28 S. Chavan, J. G. Vitillo, D. Gianolio, O. Zavorotynska, B. Civalleri, S. Jakobsen, M. H. Nilsen, L. Valenzano, C. Lamberti, K. P. Lillerud and S. Bordiga, Phys. Chem. Chem. Phys., 2012, 14, 1614-1626.

29 X. Chen, Y. Lyu, Z. Wang, X. Qiao, B. C. Gates and D. Yang, ACS Catal., 2020, 10, 2906-2914.

30 C. Xu, Y. Pan, G. Wan, H. Liu, L. Wang, H. Zhou, S.-H. Yu and H.-L. Jiang, J. Am. Chem. Soc., 2019, 141, 19110-19117. 